\title{
Increased cAMP Signaling Can Ameliorate the Hypertensive Condition in Spontaneously Hypertensive Rats
}

\author{
Torill Berg $^{\mathrm{a}}$ Eva Degerman ${ }^{\mathrm{c}}$ Kjetil Tasken ${ }^{\mathrm{b}}$ \\ ${ }^{a}$ Department of Physiology, Institute of Basic Medical Sciences and biotechnology Center, University of Oslo, \\ Oslo, Norway; 'Biomedical Center, Department of Experimental Medical Science, Lund University, Lund, Sweden
}

\section{Key Words}

Hypertension - Total peripheral vascular tension - Blood pressure $\cdot \mathrm{CAMP} \cdot$ Phosphodiesterases $\cdot \mathrm{G}_{i} \cdot$ Nitric oxide

\begin{abstract}
Background/Aim: Augmented adrenergic control of total peripheral vascular resistance (TPVR) in spontaneously hypertensive rats (SHR) may result from deficiencies in the vasodilatory system(s). Here, we studied the effect of cyclic AMP (CAMP) on TPVR-baseline and adrenergic vasoconstriction in SHR and normotensive controls (WKY). Methods: Blood pressure and cardiac output were monitored in anesthetized rats, and TPVR calculated. Results: CAMP-analogue (8CPT-CAMP) and phosphodiesterase (PDE) 3 inhibitor (milrinone) reduced TPVR in both strains. $G_{i}$ inactivator (pertussis toxin) lowered TPVR but not in all SHR. $\triangle$ TPVR induced by $\alpha_{1}$-adrenoceptor agonist (phenylephrine) was reduced by 8CPT-cAMP and milrinone in both strains. They also clearly reduced the response to endogenous noradrenaline release (tyramine) in SHR but had little effect in WKY. When pertussis toxin reduced baseline, it also eliminated the tyramine TPVR response. Propranolol did not change the effect of milrinone on the phenylephrine or tyramine response. Strain-related differences in aorta, femoral arteries or skeletal muscle PDE activity (total/PDE3/PDE4) were absent. Conclusions: CAMP signaling down-stream of CAMP was functional in SHR, and opposed $\alpha_{1}$-adrenoceptor vasoconstriction in both strains.
\end{abstract}

$\mathrm{G}_{i}$ activity greatly influenced the TPVR baseline and adrenergic TPVR responses, and its activity appeared increased in SHR. Therapeutics aiming to increase signaling through this pathway may turn out to be valuable in the treatment of hypertension.

Copyright $\odot 2008$ S. Karger AG, Basel

\section{Introduction}

An elevated total peripheral vascular resistance (TPVR) is a major characteristic of essential hypertension in man and high blood pressure in spontaneously hypertensive rats (SHR). Sympathetic nervous system hyperactivity, which has been demonstrated in both man and SHR [1-3], may be an initial factor contributing to this change. An elevated adrenergic control of TPVR may result from a shift in the homeostatic balance, either due to an increase in the vasoconstrictory reaction itself, or a failure in the vasodilatory systems which normally oppose vasoconstriction. Since the sympathetic nervous system is a major player in the control of vascular tension, the ability to ameliorate an adrenergic vascular response may therefore have great impact on the progression of the disease. Nitric oxide (NO) is an important vasodilatory agent, and reduced NO bioavailability has been suggested as an etiologic factor in spontaneous hypertension [4]. However, the TPVR response to tyramine-induced nor-

\section{KARGER}

Fax +4161306 1234 E-Mail karger@karger.ch www.karger.com
(C) 2008 S. Karger AG, Basel

$1018-1172 / 09 / 0461-0025 \$ 24.50 / 0$

Accessible online at:

www.karger.com/jvr
Dr. Torill Berg

Institute of Basic Medical Science

Department of Physiology

PO Box 1103, Blindern, NO-0317 Oslo (Norway)

Tel. +47 2285 1090, Fax +47 2285 1502, E-Mail torill.berg@medisin.uio.no 
adrenaline release was clearly more enhanced by inhibition of NO synthesis in SHR than in normotensive Wistar Kyoto controls (WKY), suggesting that the contribution of NO in the TPVR homeostasis was actually enhanced in SHR [5]. Cyclic AMP (cAMP) is another important intracellular signaling substance which induces vasodilatation when produced in vascular smooth muscle cells (VSMC), and also through its activation of NO synthesis when produced in endothelial cells [6]. The level of cAMP is regulated through the control of both synthesis and degradation. Ligand binding to stimulatory and inhibitory $G$ protein $\left(G_{s}\right.$ and $\left.G_{i}\right)$-coupled receptors regulates the activity of adenylyl cyclase, which synthesizes cAMP [7]. The degradation of cAMP is controlled by cyclic nucleotide phosphodiesterase (PDE) $3 \mathrm{~A}$ and PDE4 in VSMC and by PDEs 2, 4 and 5 in endothelial cells [8-11]. The $\beta$ adrenoceptor (AR) activates adenylyl cyclase through coupling to $G_{s}$, and a failure in this signaling pathway has been suggested as one important mechanism in the development and maintenance of hypertension [6]. The use of selective inhibitors has demonstrated that increased degradation of cAMP can play a pathogenetic role, and has identified PDEs as important drug targets in several diseases $[12,13]$. However, little is known about possible changes in cAMP signaling in spontaneous hypertension.

The first objective of the present investigation was to study the efficacy of cAMP signaling in the control of TPVR and blood pressure (BP) in early hypertensive SHR compared to that in age-matched WKY controls. Since inhibitors of cAMP signaling suitable for in vivo experiments are not easily available, we first studied the response to cAMP itself, using either the PDE-resistant cAMP analogue 8CPT-cAMP, or the selective inhibitor milrinone, the latter causing accumulation of endogenous cAMP by inhibiting its degradation by PDE3. Our second objective was to study the ability of 8CPT-cAMP and accumulated endogenous cAMP to oppose an adrenergically evoked rise in TPVR. The adrenergic response was activated by direct stimulation of VSMC $\alpha_{1}$-AR using the selective agonist phenylephrine. We also activated an adrenergic response by stimulating the release of neuronal noradrenaline with tyramine. Noradrenaline, in addition to activating the $\alpha_{1}$-AR, also stimulates $\alpha_{2^{-}}$and $\beta-A R$, and through their coupling to $G_{i}$ and $G_{s}$, respectively, they may influence adenylyl cyclase and cAMP formation. The role of these two $G$ proteins was studied by the $\beta$-AR antagonist propranolol and the $G_{i}$ inactivator pertussis toxin, respectively. The results show that cAMP signaling down-stream of cAMP was not impaired in SHR, nor did PDE activity appear to be increased in this strain, and cAMP was able to oppose $\alpha_{1}$-AR vasoconstriction in both strains. The results also present evidence indicating enhanced $G_{i}$ activity in SHR, augmenting TPVR baseline and the TPVR response to endogenous noradrenaline release.

\section{Materials and Methods}

\section{Preparation of Animals}

Male SHR rats (Okamoto, SHR/NHsd strain, $287 \pm 3$ g body weight, $12.8 \pm 0.1$ weeks, $\mathrm{n}=80$ ) and age-matched WKY (Wistar Kyoto, $290 \pm 4$ g body weight, $12.6 \pm 0.1$ weeks, $\mathrm{n}=75)$ were fed on conventional rat chow diet $(0.7 \% \mathrm{NaCl})$ and allowed food and water ad libitum until the time of the experiment. The experiments were performed in accordance with institutional guidelines and after approval by the institutional ethics committee. As previously described $[14,15]$, cardiac output (CO not including coronary flow), was measured in anesthetized (nembutal $65 \mathrm{mg}$ / $\mathrm{kg}$ i.p.) rats, using a $2 \mathrm{SB}$ perivascular flow probe on the ascending aorta and a T206 Ultrasonic Transit-Time Flow meter (Transonic Systems Inc., Ithaca, N.Y., USA). The thorax was closed after surgery, but the rats remained on the respirator, and were ventilated with air. Systolic BP (SBP) and diastolic BP (DBP) were recorded through a heparinized catheter in the femoral artery, connected to a SensoNor 840 transducer (SensoNor a/s, Horten, Norway). The flow probe and transducer were coupled to an amplifier and computer to register heart rate (HR) and to calculate mean arterial $\mathrm{BP}[\mathrm{MBP}=(\mathrm{SBP}-\mathrm{DBP}) / 3+\mathrm{DBP}], \mathrm{TPVR}(=\mathrm{MBP} / \mathrm{CO})$ and stroke volume ( $\mathrm{SV}=\mathrm{CO} / \mathrm{HR})$. Body temperature was maintained by external heating, guided by a thermosensor inserted inguinally into the abdominal cavity. Drugs were dissolved in PBS (0.01 M Na-phosphate, $\mathrm{pH} 7.4,0.14 \mathrm{M} \mathrm{NaCl})$ and administered through a catheter in the femoral vein $(0.6-1.3 \mathrm{ml} / \mathrm{kg}$ bolus injections, unless otherwise indicated).

\section{Effect of 8CPT-cAMP on Baseline and Adrenergic Tension}

Response

The ability of cAMP to lower resting TPVR was tested by the PDE-resistant cAMP analogue 8-(4-chlorophenylthio) adenosine $3^{\prime}: 5^{\prime}$-cyclic monophosphate (8CPT-cAMP). This analogue was injected first at a dose of $0.3 \mathrm{mmol} / \mathrm{kg}$ then at a dose of $4 \mathrm{mmol} / \mathrm{kg}$ 10 min later. The ability of 8CPT-cAMP to counteract adrenergic vasoconstriction was tested by its effect on the TPVR response to a bolus injection containing the selective $\alpha_{1}$-AR agonist phenylephrine $(120 \mathrm{nmol} / \mathrm{kg}$, administered $2 \mathrm{~min}$ after the second 8CPTcAMP-injection). In preliminary studies on normotensive rats, this dose of phenylephrine was established to produce a strong, but not supramaximal BP response. The vasodilatory efficacy of 8CPT-cAMP was also tested by its ability to oppose the maximum TPVR response to endogenous noradrenaline release induced by a $15-\mathrm{min}$ infusion of tyramine $(1.26 \mu \mathrm{mol} / \mathrm{min} / \mathrm{kg}$ [5]). In this protocol, due to the cost of $8 \mathrm{CPT}$-cAMP, the tyramine infusion was, unlike in the protocols below, given to the same rats $15 \mathrm{~min}$ after phenylephrine, i.e. at a time when the transient TPVR response to phenylephrine was over and TPVR had returned to the same level as before phenylephrine (documented in the legends to 
figures 4 and 5). Tyramine activates the release of catecholamines exclusively from peripheral sympathetic nerves and not from the adrenals [16]. It induces an immediate but transient rise in TPVR, with the maximum TPVR response occurring after about $3 \mathrm{~min}$ [5].

\section{Effect of the PDE3-Inhibitor Milrinone on Baselines and}

Adrenergic Tension Response

The ability of endogenous cAMP to influence resting TPVR was demonstrated by inhibiting its degradation, using the selective PDE3 inhibitor milrinone (1,6-dihydro-2-methyl-6-oxo(3,4'-bipyridine)-5-carbonitrile, $1.4 \mu \mathrm{mol} / \mathrm{kg}$ [17]). PDE3 belongs to the group of PDEs which can degrade both cAMP and cGMP, although its $\mathrm{V}_{\max }$ for cAMP is 4-10 times higher than that for cGMP [18]. The influence of NO, an activator of cGMP synthesis, on the response to milrinone was therefore controlled by giving a supramaximal dose of the non-selective NO synthase inhibitor $\mathrm{N} \omega$-nitro-L-arginine methyl ester (L-NAME, $1.1 \mathrm{mmol} / \mathrm{kg}$ ) [5] 30 min before milrinone. In addition, to investigate the role of $\beta-A R$ in the control of resting CAMP formation, rats were pretreated with the nonselective $\beta$-AR antagonist propranolol $(44 \mu \mathrm{mol} / \mathrm{kg}$, administered slowly over a 20 -min period) allowing another 20 min before administering milrinone. Adrenergic vasoconstriction was subsequently activated by a bolus injection of phenylephrine as above (not in L-NAME-treated rats) or, in separate rats, by activation of endogenous noradrenaline release using tyramine as above.

\section{Effect of $G_{i}$ Protein Inhibitor on Baselines and Response to Tyramine}

Toxin from Bordetella pertussis (PTX) was used to inhibit G proteins. WKY and SHR were injected with PTX $(15 \mu \mathrm{g} / \mathrm{kg}$ body weight i.p.) $48 \mathrm{~h}$ prior to the experiments [19].

\section{Measurement of PDE Activity in WKY and SHR Organs}

Six rats of each strain were anesthetized as above. The aorta, the femoral arteries from both sides, a skeletal muscle biopsy from $\mathrm{m}$. rectus femoris, and fat tissue from the abdomen and around the testicles were extirpated and immediately frozen $\left(-80^{\circ} \mathrm{C}\right)$. The tissue samples were later thawed on ice and transferred to scintillation vials (poly Q vials, Beckman, $4^{\circ} \mathrm{C}$ ). Homogenization buffer, $50 \mathrm{mM}$ TES (N-tris(hydroxymethyl)methyl-2-aminoethanesulfonic acid), pH 7.4, $250 \mathrm{mM}$ sucrose, $1 \mathrm{~mm}$ EDTA (ethylenediaminetetraacetic acid), 0.1 mM EGTA (ethylene glycol-bis(2-aminoethylether)- $N, N, N^{\prime}, N^{\prime}$-tetraacetic acid), $1.5 \mu \mathrm{M}$ pepstatin $\mathrm{A}, 16.5$ $\mu \mathrm{M}$ Antipain ([(S)-1-carboxy-2-phenylethyl]carbamoyl-L-arginyl-L-valyl-argininal) and $23.4 \mu \mathrm{M}$ leupeptin, was added $(3 \mathrm{ml} / \mathrm{g}$ wet weight skeletal muscle, $2 \mathrm{ml} / \mathrm{g}$ wet weight adipose tissue and arteries, and $1 \mathrm{ml} / \mathrm{g}$ wet weight aorta). The tissues were minced and homogenized either using a glass-Teflon homogenizer (adipose tissue, 10 strokes on ice) or a motorized knife homogenizer (skeletal muscle, aorta and arteries; $4-6 \times 30$ s on ice). The tissue homogenates were centrifuged for $5 \mathrm{~min}$ at $4^{\circ} \mathrm{C}$ (adipose tissue $13,000 \mathrm{~g}$, skeletal muscle, aorta and arteries $10,000 \mathrm{~g}$ ). PDE activity was measured in the supernatants as previously described [20] without or in the presence of either $3 \mu \mathrm{M}$ of the PDE3 inhibitor OPC3911 (N-cyclohexyl-N-2-hydroxyethyl-4(6-(1,2-dihydro-2oxoquinolyloxy)) butyramide) or $10 \mu \mathrm{M}$ of the PDE4 inhibitor RO 20-1724 (4-(3-butoxy-4-methoxybenzyl)-2-imidazolidinone). Since PDE3 is not present in striated muscle or endothelial cells
[10], PDE3 activity in striated muscle biopsies was therefore likely to represent VSMC activity.

\section{Drugs}

OPC 3911 was a kind gift from Otsuka Pharmaceuticals Co., Tokyo, Japan. Nembutal (pentobarbital sodium) was from the National Hospital, Oslo, Norway; 8CPT-cAMP and RO20-1724 from Biolog, GmbH, Bremen, Germany, and Antipain, leupeptin and pepstatin A from Peptide Institute, Inc., Herts, UK. The remaining drugs were from Sigma Chemical Co., St. Louis, Mo., USA.

\section{Statistics}

All results are presented as mean values \pm SEM. Recorded data from the in vivo experiments were averaged every minute throughout the experiments, but every 7 th heartbeat for the nadir response to $8 \mathrm{CPT}$-cAMP and the peak response to phenylephrine. The effect of 8CPT-cAMP on baseline (including values before 8CPT-cAMP and the nadir response after the two concentrations of $8 \mathrm{CPT}$-cAMP) and tyramine (including values before and at every minute during the 15 -min tyramine infusion period) were analyzed using repeated measures analyses of variance and covariance (BMDP Statistical Software, Inc., Statistical Solutions Ltd., Cork, Ireland). These analyses were run as overall tests including all groups or all groups within each strain (the tyramine protocol), between two groups, and for each group separately, adjusting the $\mathrm{p}$ value limit for multiple tests according to Bonferroni. When the presence of significant differences or interactions was indicated for a single curve or between two curves, Student's t tests were used to locate significant responses (one-sample) and group differences (unpaired two-sample) at the peak response ( $\mathrm{p}$ value limit $\leq 0.05$ ). The peak response occurred $3 \mathrm{~min}$ into the tyramine infusion in all groups, except in the two SHR L-NAMEtreated groups and the WKY L-NAME only group, where the peak response occurred after 6 and $5 \mathrm{~min}$, respectively. The response to milrinone was evaluated using pooled data from both the phenylephrine and the tyramine protocols. This response was therefore analyzed overall by one-way ANOVA, followed by one-sample and unpaired two-sample Student's t tests to locate significant responses and differences between groups, respectively. The same methods were used to evaluate the change from before to after PTX or phenylephrine, and to evaluate the PDE-activity results. The $\mathrm{p}$ value limit was adjusted according to Bonferroni. Correlations were determined by Pearson correlation tests using a p value limit of 0.05 .

\section{Results}

\section{Effect of the cAMP Analogue 8CPT-cAMP on}

Baselines

Baseline MBP at the start of the experiment was higher in SHR than in WKY (91 \pm 8 and $65 \pm 4 \mathrm{~mm} \mathrm{Hg}$, respectively, $\mathrm{p}=0.019)$. An elevated baseline in SHR was also observed for HR (400 \pm 13 and $324 \pm 14$ beats/min, respectively) and TPVR $(4.3 \pm 0.4$ and $2.1 \pm 0.1 \mathrm{~mm} \mathrm{Hg} /$ $\mathrm{ml} / \mathrm{min}$ ), whereas CO was less $(21 \pm 1$ and $31 \pm 2 \mathrm{ml} / \mathrm{min}$, respectively) ( $\mathrm{p} \leq 0.002)$. The cell-permeable and rather 
Fig. 1. MBP (a), TPVR (b) and CO (c) before and at the nadir response to two different doses of the cAMP analogue 8CPTcAMP (bolus injections) in SHR and WKY. Baseline values prior to $8 \mathrm{CPT}$-cAMP are given in the text. Significant changes over dose were located as indicated $\left({ }^{*} \mathrm{p} \leq\right.$ 0.0016). Six SHR and 7 WKY were included.

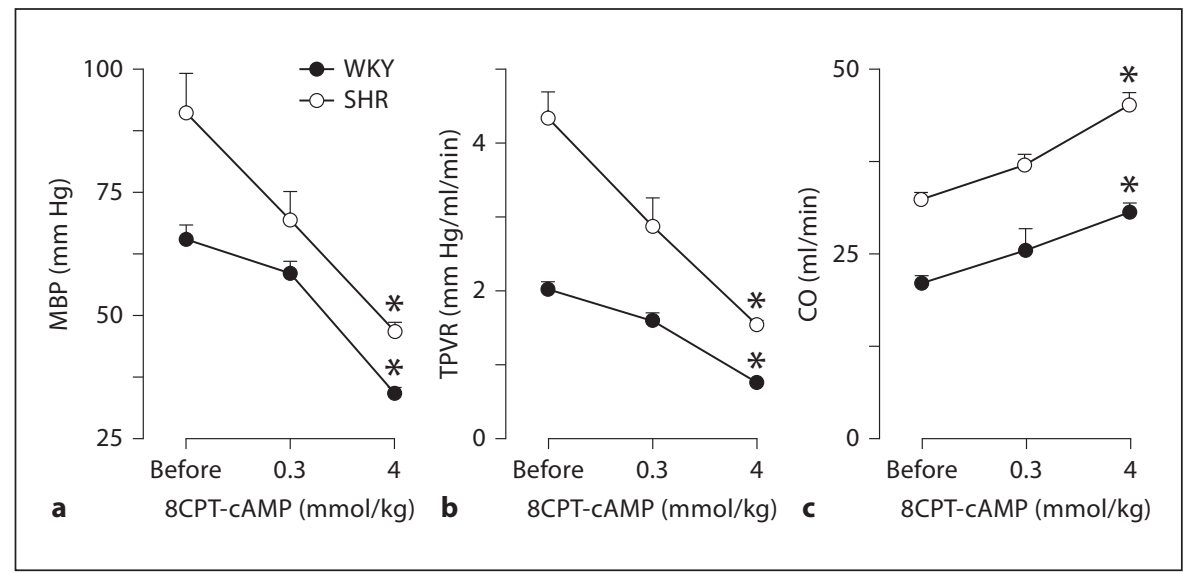

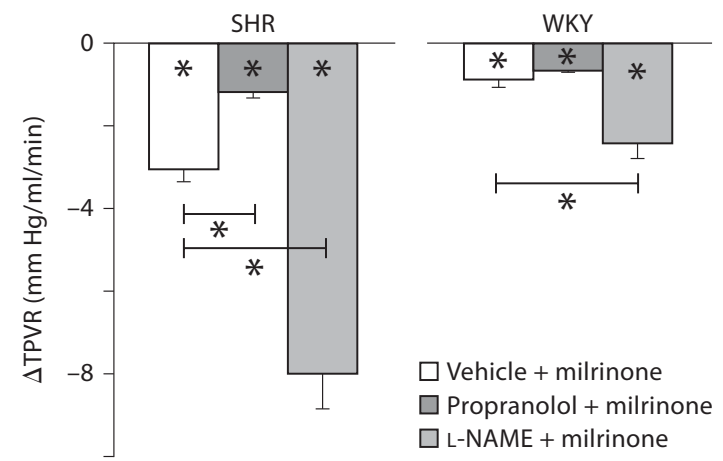

Fig. 2. The TPVR response to milrinone in SHR and WKY pretreated with vehicle (PBS) or the $\beta$-AR antagonist propranolol as indicated by the symbol legends. TPVR prior to milrinone $=5.8$ $\pm 0.3,4.0 \pm 0.3^{*}$ and $11.0 \pm 1.0^{*} \mathrm{~mm} \mathrm{Hg} / \mathrm{ml} / \mathrm{min}$ in the three SHR groups, and $2.0 \pm 0.2,2.1 \pm 0.2$ and $3.6 \pm 0.4^{*} \mathrm{~mm} \mathrm{Hg} / \mathrm{ml} /$ min in the WKY groups $(* \mathrm{p} \leq 0.006$ compared to the vehicle + milrinone group). Significant responses (* within columns) and group differences within each strain (brackets) were located as indicated. The number of rats in the six groups was $12,11,6,12$, 11 and $6 .{ }^{*} \mathrm{p} \leq 0.006$.

PDE-resistant cAMP analogue 8CPT-cAMP reduced MBP and TPVR in both strains ( $\mathrm{p} \leq 0.0003$ ) (fig. 1a, b). The response development of MBP and TPVR over concentration demonstrated that the reductions in MBP and TPVR were dose-dependent in both strains ( $\mathrm{p} \leq 0.0005$ ). $8 \mathrm{CPT}$-cAMP in addition increased CO in a dose-dependent manner ( $p \leq 0.0016$ ) (fig. 1c). There was also a slight- ly delayed dose-dependent $(\mathrm{p} \leq 0.001)$ rise in $\mathrm{HR}(\Delta \mathrm{HR}=$ $35 \pm 4$ and $8 \pm 3$ beats/min recorded 2 min after the low dose in SHR and WKY, respectively, $p=0.001$ and NS, one-sample Student's t tests, and $79 \pm 5$ and $116 \pm 10$ beats/min, respectively, for the high dose). These experiments demonstrated that SHR in the resting condition was fully able to respond to cAMP with vasorelaxation.

\section{Effect of the PDE3-Inhibitor Milrinone on Baselines}

Similar to that seen for the cAMP analogue, accumulation of endogenous CAMP, induced by inhibition of its degradation with the PDE3 inhibitor milrinone, lowered MBP (from $108 \pm 6$ to $41 \pm 4 \mathrm{~mm} \mathrm{Hg}$ in SHR and from $61 \pm 5$ to $30 \pm 3 \mathrm{~mm} \mathrm{Hg} \mathrm{WKY,} \mathrm{respectively,} \mathrm{p}<0.001$ for effect of milrinone and strain-related differences). Milrinone also reduced TPVR, and with a greater effect in SHR than in WKY (fig. 2) ( $<<0.001)$, although TPVR after milrinone was still higher in SHR than in WKY (2.7 \pm 0.2 and $1.1 \pm 0.1 \mathrm{~mm} \mathrm{Hg} / \mathrm{ml} / \mathrm{min}$ in SHR and WKY, respectively, $\mathrm{p}<0.001)$. A strong correlation was seen between baseline TPVR and the $\triangle$ TPVR in response to milrinone $(c=-0.84$ and -0.79 in SHR and WKY, respectively, $\mathrm{p} \leq 0.002)$. The PDE3 inhibitor had no effect on HR in either strain $(\Delta \mathrm{HR}=-4 \pm 2$ and $-3 \pm 2 \%$ in SHR and WKY, respectively, $\mathrm{p}=\mathrm{NS})$. Neither was there any effect on CO or SV in WKY ( $<7 \%, \mathrm{p}=\mathrm{NS})$, but CO and SV were lowered by $20 \pm 5$ and $17 \pm 4 \%$, respectively, in SHR $(p=0.002)$. These results demonstrated that inhibition of cAMP degradation, with the subsequent increase in the concentration of endogenous cAMP, reduced baseline TPVR in both strains.

The $\beta_{1+2}$-AR antagonist propranolol lowered TPVR baseline in SHR only (fig. 2, legend). A comparable reduction was seen in the TPVR-response to milrinone in 
Fig. 3. Typical MBP, HR, CO and TPVR traces from SHR and WKY given a sham injection containing PBS, followed $10 \mathrm{~min}$ later by a bolus injection of phenylephrine (left panel) or a 15-min infusion with tyramine (right panel).

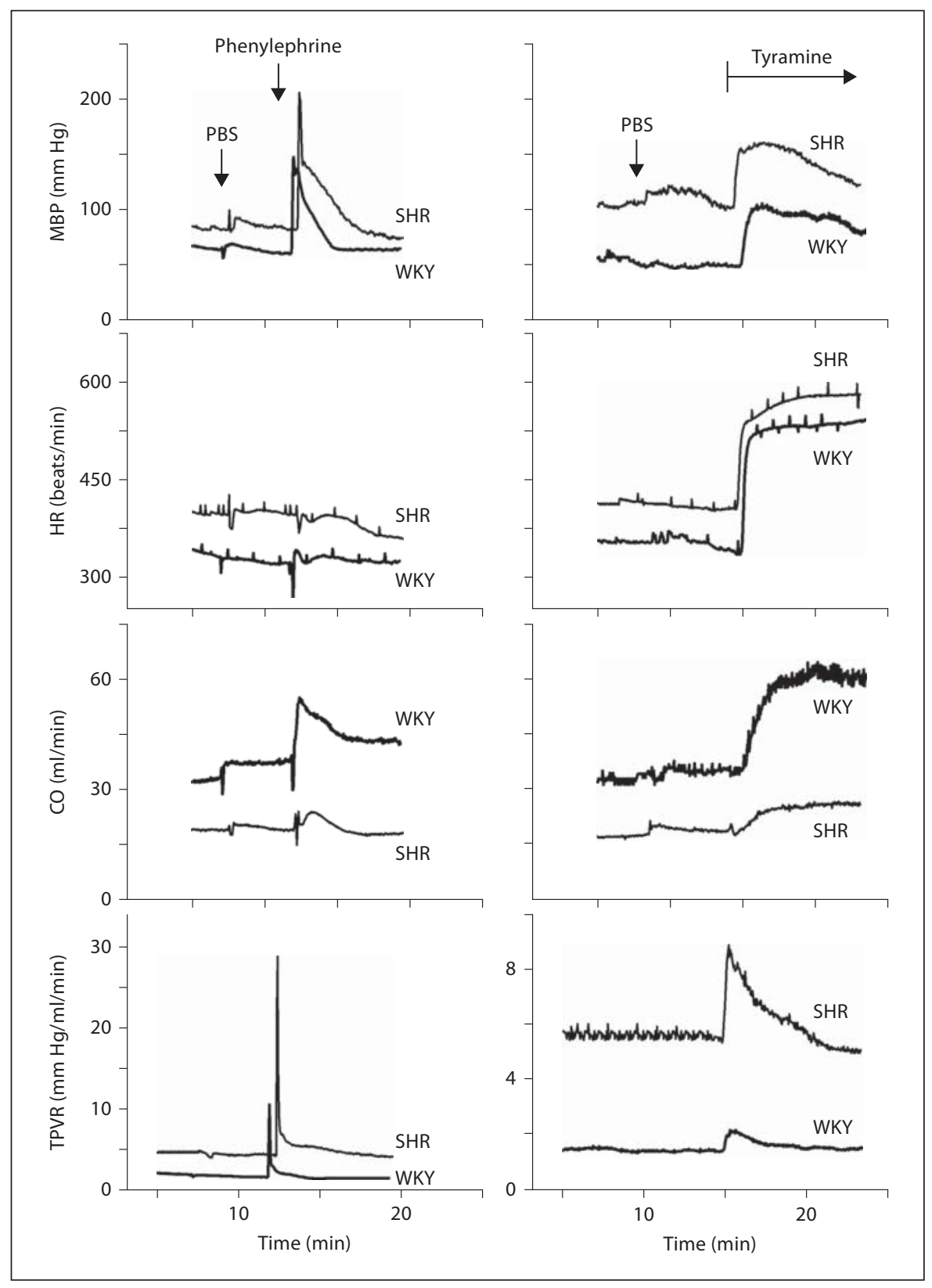

propranolol-treated SHR ( $\mathrm{p}<0.001)$, whereas propranolol had no effect on the response to milrinone in WKY $(\mathrm{p}=\mathrm{NS}$ ) (fig. 2). After pretreatment with the nonselective NOS inhibitor L-NAME, the TPVR response to the PDE3 inhibitor was augmented by 2.6 and 2.7 times in SHR and WKY, respectively ( $\mathrm{p} \leq 0.001$ ) (fig. 2). The effect of LNAME on baseline (fig. 2, legend) resembled in magnitude its effect on the TPVR response to milrinone. The strong negative correlation between TPVR prior to milrinone and $\triangle \mathrm{TPVR}$ in response to milrinone remained when both strains and the propranolol and L-NAME pretreatments were included $(c=-0.97, \mathrm{p}<0.001)$.

\section{Effect of the $G_{i}$-Inhibitor PTX on Baselines}

TPVR baseline was reduced by PTX in both strains (3.4 \pm 0.6 and $5.0 \pm 0.3 \mathrm{~mm} \mathrm{Hg} / \mathrm{ml} / \mathrm{min}$, in the PTX-treated and control SHR groups, respectively, $\mathrm{p}=0.015$, and 1.4 \pm 0.2 and $1.9 \pm 0.2 \mathrm{~mm} \mathrm{Hg} / \mathrm{ml} / \mathrm{min}$ in WKY, $\mathrm{p}=0.045$ ). When the TPVR response in SHR was separated into responders and nonresponders, TPVR was clearly reduced 


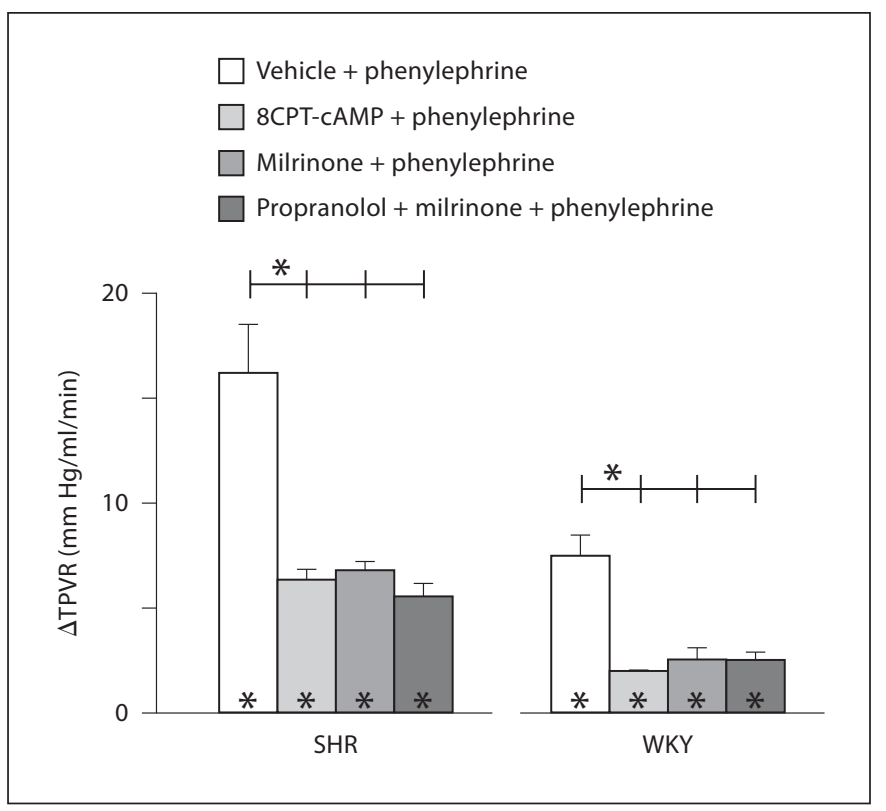

Fig. 4. The peak TPVR response to the $\alpha_{1}$-AR agonist phenylephrine in SHR and WKY after pretreatment with the PDE-resistant cAMP analogue 8CPT-cAMP and the PDE3 inhibitor milrinone, as indicated by symbol legends. TPVR prior to phenylephrine $=$ $5.8 \pm 0.4,3.2 \pm 0.2^{*}, 2.1 \pm 0.1^{*}$ and $2.1 \pm 0.3^{*} \mathrm{~mm} \mathrm{Hg} / \mathrm{ml} / \mathrm{min}$ in the four SHR groups, and $2.4 \pm 0.2,1.1 \pm 0.1^{*}, 1.0 \pm 0.2^{*}$ and $0.8 \pm 0.1^{*} \mathrm{~mm} \mathrm{Hg} / \mathrm{ml} / \mathrm{min}$ in the four WKY groups $\left({ }^{*} \mathrm{p} \leq 0.001\right.$ compared to the controls groups). Significant responses (* within columns) and group differences within each strain (brackets) were detected as indicated. The number of rats in the eight groups was $10,6,6,5,13,7,6$ and 5 , respectively. ${ }^{*} \mathrm{p} \leq 0.006$.

in 6 of the 9 rats tested (TPVR $=2.3 \pm 0.6 \mathrm{~mm} \mathrm{Hg} / \mathrm{ml} /$ min, $\mathrm{p}<0.001$ ), but not at all different in the 3 remaining rats $(\mathrm{TPVR}=5.4 \pm 0.7 \mathrm{~mm} \mathrm{Hg} / \mathrm{ml} / \mathrm{min}, \mathrm{p}=\mathrm{NS})$. TPVR after PTX was greater in SHR than WKY, even in the 6 responding SHR $(\mathrm{p}=0.009)$. PTX reduced baseline MBP (67 \pm 6 and $85 \pm 5 \mathrm{~mm} \mathrm{Hg}$ with and without PTX, respectively, $\mathrm{p}=0.034)$ in SHR, whereas the difference in MBP was not statistically significant in WKY. HR was higher in PTX-treated rats of both strains $(395 \pm 11$ and $354 \pm 8$ beats/min with and without PTX in SHR, and $346 \pm 10$ and $309 \pm 11$, respectively, in WKY, $\mathrm{p} \leq 0.029)$. PTX had no significant effect on CO (data not shown).

\section{Effect of 8CPT-cAMP and Milrinone on the TPVR}

Response to Phenylephrine

Selective activation of postsynaptic $\alpha_{1}$-AR, using a bolus injection of phenylephrine, induced a sharp, but transient rise in MBP and TPVR (fig. 3, left panel). Both re- sponses were greater in SHR than in WKY $(\triangle \mathrm{MBP}=102$ \pm 8 and $82 \pm 2 \mathrm{~mm} \mathrm{Hg}$, respectively, $\mathrm{p}=0.027$, and $\Delta \mathrm{TPVR}=16.2 \pm 2.3$ and $7.5 \pm 1.0 \mathrm{~mm} \mathrm{Hg} / \mathrm{ml} / \mathrm{min}, \mathrm{p}=$ 0.006; fig. 4). A concomitant reduction ( $\mathrm{p} \leq 0.001)$ was detected in $\mathrm{CO}(\Delta \mathrm{CO}=-38 \pm 8$ and $-32 \pm 5 \%$ in SHR and $\mathrm{WKY}$, respectively). However, the changes in HR were negligible $(\Delta \mathrm{HR}=-4 \pm 1 \%, \mathrm{p}=0.001$, in SHR, and $1 \pm 3 \%, \mathrm{p}=\mathrm{NS}$, in WKY), demonstrating that baroreceptor reflexes were not activated in these anesthetized rats. Typical traces of the CO- and HR-responses are shown in figure 3.

Pretreatment with $8 \mathrm{CPT}$-cAMP or the PDE3 inhibitor milrinone clearly reduced the phenylephrine-induced $\alpha_{1}$-adrenergic TPVR response in both strains $(\mathrm{p} \leq 0.005)$ (fig. 4). This observation demonstrated the ability of exogenous and endogenous cAMP to ameliorate an $\alpha_{1}$-adrenergic vasoconstriction. $\triangle \mathrm{TPVR}$ to phenylephrine remained clearly greater in SHR than in WKY also after milrinone (fig. 4). The TPVR-response to phenylephrine after additional pre-treatment with the $\beta$-AR antagonist propranolol prior to milrinone was not different from that after milrinone alone in either strain ( $\mathrm{p}=\mathrm{NS})$ (fig. 4). Thus, the accumulated cAMP, responsible for counteracting the response to the $\alpha_{1}$-AR agonist, did not depend on $\beta$-AR activation for its synthesis.

\section{Effect of 8CPT-cAMP, Milrinone and PTX on the \\ TPVR Response to Tyramine}

As previously documented [5], activation of endogenous noradrenaline release, using a 15-min infusion with tyramine, induced an immediate but transient rise in TPVR, which was 4.6 times greater in SHR than in WKY $(\mathrm{p}<0.001)$ (fig. 5). Tyramine also induced a tachycardia for the duration of the infusion period ( $\mathrm{p}=0.001$, from $348 \pm 9$ beats/min before tyramine to $492 \pm 11$ beats/ min at the end of the 15-min infusion period in SHR, and from $306 \pm 12$ to $493 \pm 12$ beats/min in WKY). Typical traces of the MBP, CO, HR and TPVR responses are shown in figure 3 (right panel). We detected no correlation between the TPVR peak response to tyramine and TPVR baseline in the SHR and WKY control groups ( $\mathrm{n}=$ 19 and 13 rats, respectively).

When tyramine was infused into the 8CPT-cAMPtreated rats, the initial rise in TPVR was reduced to approximately half in SHR ( $\mathrm{p}=0.002)$, whereas no significant effect was observed in WKY (fig. 5). Similarly, the accumulation of endogenous cAMP induced by pretreatment with milrinone totally abolished the TPVR response to tyramine in SHR ( $\mathrm{p}=\mathrm{NS}$ and $<0.001$, one- and twosample Student's t tests, respectively) and also caused a 


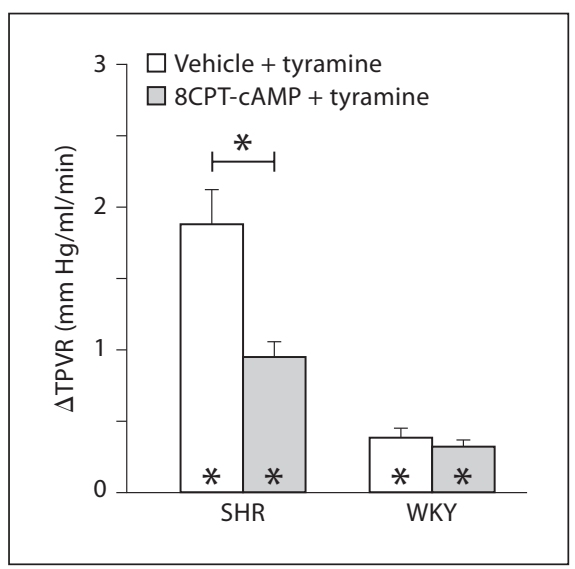

Fig. 5. The TPVR peak response to tyramine in SHR and WKY after pretreatment with the PDE-resistant cAMP analogue 8CPTcAMP. TPVR prior to tyramine $=5.1 \pm 0.3$ and $3.4 \pm 0.2 \mathrm{~mm}$ $\mathrm{Hg} / \mathrm{ml} / \mathrm{min}$ in the two SHR groups $(\mathrm{p}<0.001)$, and $1.9 \pm 0.2$ and $1.2 \pm 0.1 \mathrm{~mm} \mathrm{Hg} / \mathrm{ml} / \mathrm{min}$ in the two WKY groups $(\mathrm{p}<0.001)$. Significant responses ( ${ }^{*}$ within columns) and group differences within each strain (brackets) were located as indicated. The number of rats was 19, 6, 13 and 7 in the four groups, respectively. ${ }^{*} \mathrm{p} \leq 0.008$.

small reduction in $\triangle \mathrm{TPVR}$ in WKY $(\mathrm{p}=0.038$ and 0.036 , respectively) (fig. 6). The PDE3 inhibitor had no effect on the tachycardia following tyramine in either strain; at the end of the 15 min infusion $\Delta \mathrm{HR}=153 \pm 12$ and $141 \pm$ 7 beats/min with and without milrinone, respectively, in SHR, and $175 \pm 9$ and $187 \pm 9$ beats/min in WKY ( $\mathrm{p}=$ NS in both strains). The effect of milrinone on the TPVRresponse to tyramine was not changed in either strain by administration of the $\beta_{1+2}$-AR antagonist propranolol prior to milrinone (fig. 6). Thus, similar to that seen for phenylephrine, the accumulated cAMP after PDE3 inhibition, counteracting the TPVR response to neuronal noradrenaline release, did not depend on $\beta$-AR activity for its synthesis.

Milrinone clearly reduced the augmented TPVR response to tyramine following inhibition of NO-induced cGMP formation by the NO synthase inhibitor L-NAME in both strains (fig. 6). The ameliorating effect of milrinone was therefore not due to inhibition of PDE3-induced cGMP-degradation. The TPVR-response to tyramine after L-NAME+milrinone was lower than that in the controls $(\mathrm{p}=0.004)$ and higher than that after milrinone alone in SHR ( $p=0.011)$, but not in WKY (fig. 6).

Inhibition of $G_{i}$ with PTX reduced the TPVR response to tyramine in SHR $(p=0.009)$ and totally eliminated the response in WKY ( $\mathrm{p}=0.002$ and NS, two- and one-sample

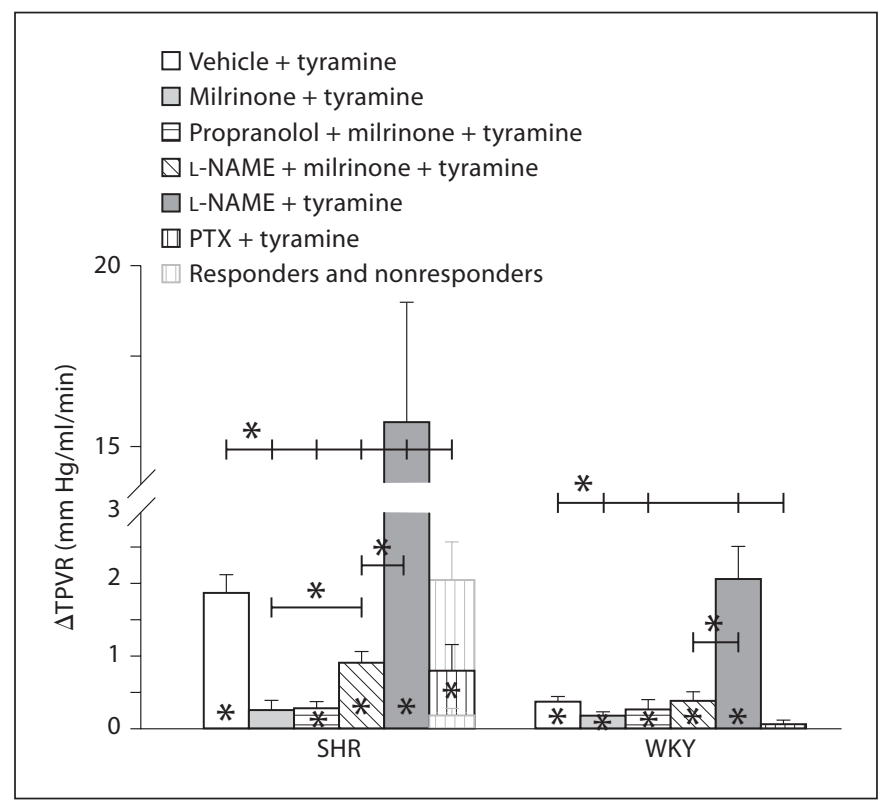

Fig. 6. The effect of the PDE3 inhibitor milrinone or PTX on the TPVR peak response to tyramine in SHR and WKY. Milrinone was given alone or combined with the $\beta$-AR antagonist propranolol or the NO synthase inhibitor L-NAME as indicated by symbol legends. The response to tyramine after L-NAME alone has previously been published in [5]. The responses to tyramine in PTXtreated SHR were divided into responders and nonresponders, dependent on whether PTX reduced or did not reduce, respectively, baseline TPVR and the response to tyramine. These are indicated by the faded grey columns, whereas the mean is shown in black. TPVR prior to tyramine $=5.0 \pm 0.3,3.2 \pm 0.2^{*}, 3.3 \pm$ $0.2^{*}, 3.0 \pm 0.3^{*}, 11.3 \pm 1.5^{*}$ and $3.4 \pm 0.6 \mathrm{~mm} \mathrm{Hg} / \mathrm{ml} / \mathrm{min}$ in the six SHR groups, and $1.9 \pm 0.2,1.2 \pm 0.1^{*}, 1.2 \pm 0.1^{*}, 1.2 \pm 0.1^{*}$, $2.8 \pm 0.3$ and $1.4 \pm 0.2^{*} \mathrm{~mm} \mathrm{Hg} / \mathrm{ml} / \mathrm{min}$ in the six WKY groups (* indicating $\mathrm{p} \leq 0.009$ compared to the corresponding control). After repeated measures analyses of variance and covariance had demonstrated differences or interaction over time between specific group or for separate groups within each strain ( $p$ value limit $\leq 0.0083$ and 0.01 , respectively), significant responses $\left({ }^{*}\right.$ within columns) and group differences (brackets) at the peak response were detected as indicated. The number of rats in the six SHR groups was 18, 6, 6, 6 and 9, and in the six WKY groups 13, 6, 6, 6 and $5 .^{*} \mathrm{p} \leq 0.05$.

Student's t tests, respectively) (fig. 6). In SHR, the response was not reduced in the three rats where PTX did not lower baseline (nonresponders) ( $\mathrm{p}=\mathrm{NS}$ ), whereas the response was totally eliminated in the remaining six where PTX clearly reduced baseline (responders) ( $\mathrm{p}=\mathrm{NS}$ and 0.024, one- and two-sample Student's t tests, respectively) (fig. 6). PTX also reduced the MBP and CO response to tyramine in both strains ( $p \leq 0.0088$ ), but had little effect on the tyramine-induced tachycardia (data not shown). 
Fig. 7. PDE activity measurements in tissue homogenates. Total PDE (left panel), PDE3 and PDE4 activity (right panel) was measured in homogenates from aorta, arteries and skeletal muscle. Significant strain- and organ-related differences were not detected.

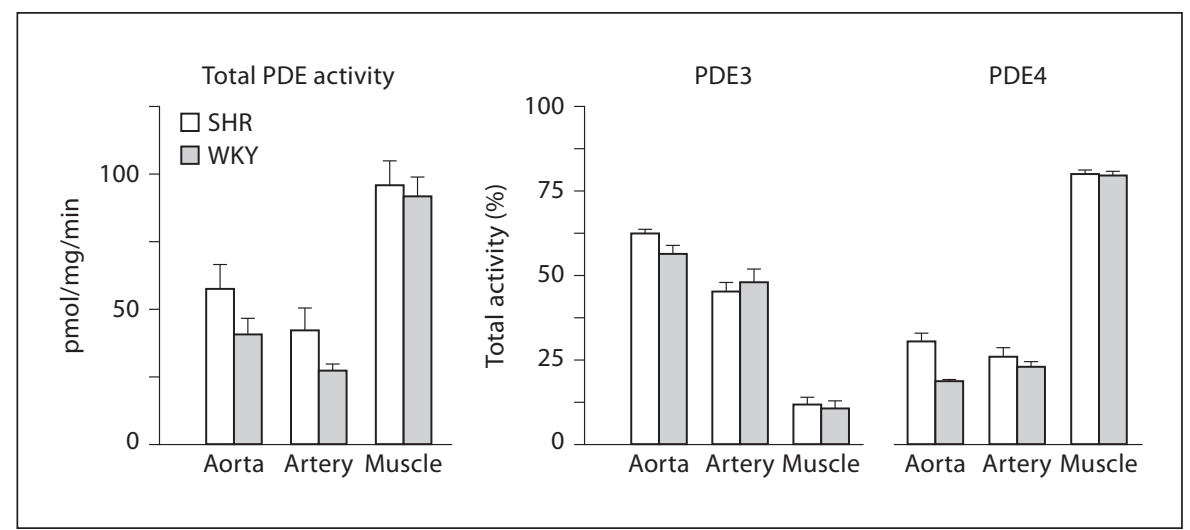

\section{Measurement of PDE Activity in SHR and WKY} Organs

Total PDE activity in aorta, femoral arteries and striated muscle in SHR was not significantly different from that measured in WKY (fig. 7). 71-93\% of the total activity in aorta and the arteries represented PDEs 3 and 4 . In both organs and in both strains, the activity of PDE3 was approximately twice that of PDE4 ( $\mathrm{p} \leq 0.001)$. There was no strain- or organ-related difference in the relative PDE3 and PDE4 activities either (fig. 7). Neither were strainrelated differences in total or subtype PDE activity in the muscle biopsies detected, but in muscle the level of PDE4 activity was about 7 times that of the PDE3 (fig. 7), the latter representing enzyme activity from VSMC within the striated muscle. We also did not detect a strain-related difference $(\mathrm{p}=\mathrm{NS})$ in PDE activity in abdominal fat $(63 \pm 7,33 \pm 5$ and $17 \pm 4 \mathrm{pmol} / \mathrm{mg} / \mathrm{min}$, respectively, for total, and 3- and 4-subtype PDE activity in SHR, and $51 \pm 7,26 \pm 5$ and $16 \pm 2 \mathrm{pmol} / \mathrm{mg} / \mathrm{min}$, respectively, in WKY) or testicular fat $(79 \pm 4,42 \pm 2$ and $22 \pm 3$ $\mathrm{pmol} / \mathrm{mg} / \mathrm{min}$ in SHR, and $75 \pm 6,42 \pm 4$ and $19 \pm 4$ $\mathrm{pmol} / \mathrm{mg} / \mathrm{min}$, respectively, in WKY). These results demonstrated that PDE activity, total and the 3 and 4 subtypes, was not altered in the SHR compared to WKY tissues.

\section{Discussion}

In the present study, we detected no impairment in the ability of cAMP to lower TPVR in the SHR, as tested with the analogue 8CPT-cAMP or accumulation of endogenous cAMP using the PDE3 inhibitor milrinone. Moreover, PDE activity in the vasculature was not increased in this strain. We also found that 8CPT-cAMP and the
PDE3 inhibitor efficiently reduced the TPVR response to direct stimulation of VSMC $\alpha_{1}$-AR with phenylephrine in both strains. However, these two agents greatly ameliorated the rise in TPVR following tyramine-induced transmitter release from peripheral sympathetic nerves in the SHR, but had no or little effect on the much smaller TPVR response to tyramine in WKY. Furthermore, inhibition of $G_{i}$ with PTX reduced baseline TPVR and totally abolished the TPVR response to tyramine in all WKY rats. In SHR, the inhibitory effect of $\mathrm{G}_{\mathrm{i}}$ was not so easily overcome by PTX, where baseline TPVR and the response to tyramine remained intact in $1 / 3$ of the rats.

The PDE-resistant cAMP analogue 8CPT-cAMP elicited a dose-dependent fall in MBP due to a reduction in TPVR in both strains, in that way demonstrating the ability of cAMP to induce systemic vasodilatation. Similarly, a reduction in baseline TPVR was also seen in response to the selective PDE3 inhibitor milrinone in both strains. Although locating the cell and mechanism responsible for a complicated homeostatic mechanism such as blood pressure control in whole animal experiments is a challenge, we may conclude that the hypotensive effect of 8CPT-cAMP and milrinone was not likely to result from a reduction in the release of noradrenaline, since cAMP has been shown to increase noradrenaline release [21]. Furthermore, since PDE3 is one of the two main PDEs in VSMC but is not present in the endothelium [8, 10], the milrinone-induced fall in TPVR most likely resulted from an accumulation of CAMP in the VSMC. We therefore concluded that 8CPT-cAMP and milrinone induced vasodilatation by activating cAMP signaling within the VSMC.

The TPVR response to both 8CPT-cAMP and milrinone was greater in SHR than in WKY. However, since TPVR is inversely proportional to the fourth power of the 
resistance artery internal radius (Poiseuille's law), it could, from the elevated TPVR baseline in SHR, be deduced that the vessel lumen diameter was less in this strain. Thus, any vasoconstrictory or vasodilatory stimulus will, according to Poiseuille's law, have a greater impact on TPVR in SHR. The enhanced response to 8CPTcAMP and milrinone in SHR compared to WKY was therefore likely, at least in part, to be explained by the elevated baseline TPVR in SHR. From these results, we concluded that VSMC signaling through the CAMP pathway, downstream of cAMP, was not impaired in the SHR.

A change in the lumen diameter may result from vessel wall remodeling or from differences in the vasoconstrictory tone. In SHR, the resistance arteries have been shown to undergo predominantly inward eutrophic remodeling [22], resulting in a reduced internal diameter. Since this remodeling has been shown to occur as early as in 8-week-old rats [23], similar changes were likely to be present in our 12- to 14-week-old rats. The remodeling of the resistance arteries in SHR will therefore influence comparisons made across the two strains. However, comparisons within each strain separately will reflect changes in the vasoconstrictory or vasodilatory components controlling resistance vessel diameter and TPVR, independent of structural remodeling. Still, from Poiseuille's law, changes in baseline induced by one agent will have a significant impact on the change in TPVR in response to a subsequent stimulus. Differences in baseline TPVR were therefore likely to explain the differences in the TPVR response to milrinone within each strain after pretreatment with L-NAME or propranolol. This conclusion was supported by the observation that the difference in the TPVR response to milrinone after L-NAME was comparable to the effect of L-NAME on TPVR baseline. The same was true for the reduced TPVR response to milrinone after the propranolol-induced reduction in TPVR baseline in SHR. Moreover, the TPVR response to milrinone was strongly and negatively correlated with TPVR baseline both in the two milrinone control groups separately and in all groups of both strains pooled. PDE3 also degrades cGMP, and through its competition with cAMP, cGMP is often referred to as an inhibitor of PDE3 [13,24]. However, the cGMP inhibition of PDE3 appeared negligible in these in vivo experiments, in agreement with that the PDE3 $V_{\max }$ for cAMP is $4-10$ times higher than that for cGMP [18]. Moreover, TPVR after milrinone, propranolol+milrinone or L-NAME+milrinone did not differ within each strain, indicating that the milrinone-induced accumulation of cAMP fully relaxed the VSMC in both strains, although with a strain-related difference in TPVR remaining.

It may be hypothesized that the elevated TPVR baseline in SHR results from an increased degradation of VSMC cAMP. However, we detected no difference between the two strains in the total PDE-activity or the concentration of PDE3 and PDE4 in extirpated aorta and femoral arteries. We also found no strain-related difference in the concentration of PDE3 in striated muscle biopsies. Striated muscle cells contain PDE4, but neither striated muscle nor endothelial cells contain the PDE3 subtype [10]. The PDE3 activity in striated muscle therefore represented PDE3 in VSMC, and was perhaps the most reliable measurement of this enzyme in VSMC. This biopsy was not contaminated with fat tissue, whereas the large vessels had to be carefully dissected from surrounding fat, which is rich in PDE3B [25]. A strain-related difference in the PDE activities in abdominal or testicular fat tissue was also not detected. In addition, the striated muscle biopsy would contain mostly small resistance vessels, whereas aorta and femoral arteries are conduit vessels which are largely not involved in determining TPVR. From these observations we concluded that increased degradation of CAMP did not explain the elevated baseline TPVR in the SHR.

The ability of cAMP to mollify $\alpha_{1}$-AR vasoconstriction was demonstrated in the present study by the observation that both $8 \mathrm{CPT}$-cAMP and milrinone greatly reduced the TPVR response to phenylephrine, and they did so clearly in both strains with a strain-related difference remaining. The reductions in the response to phenylephrine were to a large extent paralleled by the 8CPTcAMP- and milrinone-induced reductions in baseline. For instance, a 64 and 58\% reduction in TPVR baseline in SHR and WKY, respectively, resulted in a 58 and $56 \%$ reduction in the TPVR response to phenylephrine. Thus, the effect of cAMP on the TPVR response to phenylephrine appeared to depend largely on the Poiseuille lumen-resistance relationship, as discussed above. However, when vasoconstriction was activated by tyramine, the modulating role of cAMP differed in the two strains: 8CPT-cAMP almost halved and milrinone totally eliminated the TPVR response to tyramine in SHR, whereas these two agents had no and little effect, respectively, on the much smaller TPVR response to tyramine in WKY. Since the effect of 8CPT-cAMP and milrinone on TPVR baseline was the same in the two protocols, the different effect of these two agents on the response to phenylephrine and tyramine could not be explained by differences in baselines. Moreover, the TPVR response to tyramine 
after milrinone no longer differed between the two strains, although a difference in baseline remained. Thus, the $83 \%$ reduction in the response to tyramine after the 36\% milrinone-induced reduction in TPVR baseline in SHR could not be explained in full by Poiseuille's law.

Poiseuille's law was also not likely to explain why SHR were less responsive to PTX than WKY. PTX reduced TPVR baseline and eliminated the TPVR response to tyramine in all WKY rats, whereas $1 / 3$ of the 9 rats in the SHR group was not influenced by PTX. However, when PTX reduced TPVR baseline in SHR, it, like milrinone, also almost totally eliminated the response to tyramine. These observations again suggested a critical role of cAMP in the control of baseline TPVR and through that the magnitude of the response to $\alpha_{1}$-AR vasoconstriction. In addition, inhibition of $G_{i}$ or accumulation of cAMP had a greater impact on the TPVR response to tyramine than phenylephrine. Since PTX increased HR baseline but had only minor effects on the tyramine-induced tachycardia, the reduced TPVR baseline and tyramine response were not likely to be due to a reduction in noradrenaline release. However, phenylephrine elicits a vasoconstrictory response by a direct effect on VSMC $\alpha_{1^{-}}$ $A R$, whereas tyramine activates peripheral, neuronal noradrenaline release by reverse transport through the reuptake-1 mechanism [26]. Thus, the present observations appeared to indicate that the difference between the two strains involved the release of noradrenaline and/or cotransmitters and their role in cAMP formation, i.e. upstream of the cAMP-effector mechanisms. Noradrenaline, unlike phenylephrine, also activates $\alpha_{2}$-ARs, which couple to $G_{i}$ and inhibit adenylyl cyclase and, hence, cAMP formation. Based on the reduced sensitivity to PTX in SHR, $G_{i}$ inhibition of VSMC adenylyl cyclase seemed enhanced in SHR. This conclusion was supported by the observed delay in the development of hypertension in SHR after inhibition of $G_{i}$ [27]. An augmented effect of $G_{i}$ during noradrenaline release may be due to altered function of the $G_{i}$ protein itself: polymorphism in the G-protein $\beta_{3}$ subunit gene, with increased signaling through the $G_{i}$ pathway, has been linked to human hypertension [28]. However, the reduced efficiency of PTX in overcoming the effect of $G_{i}$ on TPVR baseline in SHR may also result from an increased sympathetic activity and $\alpha_{2}-A R-G_{i}$ signaling in this strain, where central sympathetic output has been shown to be greater than in WKY [29]. In agreement with the present results, such signaling will be further activated during stimulated noradrenaline release.
Inefficient signaling through the $\beta-A R-G_{s}$ pathway has also been suggested as a pathogenic factor in hypertension, either due to deficiencies in the $\beta$-AR itself, or from reduced uptake of circulating adrenaline and its corelease with noradrenaline [30]. However, we found no effect of the $\beta$-AR antagonist propranolol on the effect of milrinone on baseline TPVR in SHR or WKY other than that which could be explained by Poiseuille's law. We also did not observe any effect of propranolol on the ameliorating effect of milrinone on the TPVR response to phenylephrine or tyramine in either strain. We therefore concluded that under the present conditions, activation of VSMC $\beta$-AR did not influence adenylyl cyclase activity and cAMP formation at rest or during the TPVR peak response to phenylephrine or tyramine. Thus, deficiencies in the $\beta$-AR VSMC cAMP formation were not responsible for the elevated TPVR baseline or the augmented adrenergic TPVR response in SHR.

8CPT-cAMP has been shown to increase HR [31]. This observation was paralleled by our present results. However, milrinone had no effect on baseline HR, nor did milrinone alter the tachycardia induced by tyramine. This differed from the inhibitory effect of milrinone on the sympathetic-mediated tachycardia previously observed in the pithed rat [17], and we have no explanation for this difference. Furthermore, in the present experiments, we used rats anesthetized with pentobarbital. This prevented activation of baroreceptor reflexes, as clearly demonstrated by the finding that bradycardia was not elicited during the high rise in BP in response to phenylephrine. We therefore concluded that cardiovascular reflexes were not likely to influence the BP and TPVR responses studied and interfere with the results.

In conclusion, the present study clearly demonstrated the importance of the cAMP pathway in the control of baseline TPVR and BP, and, through that, its effect on the vasoconstrictory response to adrenergic activation. cAMP signaling in the VSMC, downstream of cAMP, was not impaired in SHR, and increased degradation of cAMP by PDEs was not observed. Therefore, a failure in the cAMP effector mechanisms did not explain the elevated baseline TPVR in SHR. However, as indicated by the reduced sensitivity to PTX, a stronger inhibitory effect of $G_{i}$ on adenylyl cyclase appeared to be present in SHR. The augmented effect of $\mathrm{G}_{\mathrm{i}}$ in SHR could be circumvented by preventing cAMP degradation, in that manner allowing endogenous VSMC cAMP to accumulate. The inhibitory effect of $G_{i}$ was more pronounced when noradrenaline was stimulat- 
ed. Thus, transmitter release and a concomitant activation of $G_{i}$, possibly through activation of the $\alpha_{2}$-AR, may play a role in elevating resting TPVR and the more pronounced response to adrenergic vasoconstriction in SHR. Therapeutics aiming to increase signaling through the cAMP pathway may therefore turn out to be valuable in the treatment of high BP.

\section{Acknowledgements}

This study was supported by grants from the Norwegian Council on Cardiovascular Research (T.B.), the Swedish Medical Research Council (grant 3362) (E.D.), the Functional Genomics Programme (K.T.), the Research Council of Norway (K.T.), Novo Nordic Foundation (K.T.) and the European Union (grant 037189, thera-cAMP) (K.T.).

\section{References}

1 Rahn KH, Barenbrock M, Hausberg M: The sympathetic nervous system in the pathogenesis of hypertension. J Hypertens 1999; 17(suppl 3):S11-S14.

-2 Esler M, Rumantir M, Kaye D, Jennings G, Hastings J, Socratous F, Lambert G: Sympathetic nerve biology in essential hypertension. Clin Exp Pharmacol Physiol 2001;28: 986-989.

-3 Guyenet PG: The sympathetic control of blood pressure. Nat Rev Neurosci 2006;7: 335-346.

4 Hermann M, Flammer A, Luscher TF: Nitric oxide in hypertension. J Clin Hypertens 2006;8:17-29.

$\checkmark 5$ Berg T: Increased counteracting effect of eNOS and nNOS on an alpha(1)-adrenergic rise in total peripheral vascular resistance in spontaneous hypertensive rats. Cardiovasc Res 2005;67:736-744.

6 Feldman RD, Gros R: Defective vasodilatory mechanisms in hypertension: a G-proteincoupled receptor perspective. Curr Opin Nephrol Hypertens 2006;15:135-140.

77 Taussig R, Gilman AG: Mammalian membrane-bound adenylyl cyclases. J Biol Chem $1995 ; 270: 1-4$.

8 Polson JB, Strada SJ: Cyclic nucleotide phosphodiesterases and vascular smooth muscle. Annu Rev Pharmacol Toxicol 1996;36:403427.

-9 Degerman E, Belfrage P, Manganiello VC: Structure, localization, and regulation of cGMP-inhibited phosphodiesterase (PDE3). J Biol Chem 1997;272:6823-6826.

10 Ashikaga T, Strada SJ, Thompson WJ: Altered expression of cyclic nucleotide phosphodiesterase isozymes during culture of aortic endothelial cells. Biochem Pharmacol 1997;54:1071-1079.

-11 Bender AT, Beavo JA: Cyclic nucleotide phosphodiesterases: molecular regulation to clinical use. Pharmacol Rev 2006;58:488520
12 Houslay MD, Schafer P, Zhang KY: Keynote review: phosphodiesterase- 4 as a therapeutic target. Drug Discov Today 2005;10:15031519.

13 Matsumoto T, Kobayashi T, Kamata K: Phosphodiesterases in the vascular system. J Smooth Muscle Res 2003;39:67-86.

14 Berg T: Analysis of the pressor response to the $\mathrm{K}(+)$ channel inhibitor 4-aminopyridine. Eur J Pharmacol 2002;452:325-337.

15 Berg T: The vascular response to the $\mathrm{K}+$ channel inhibitor 4-aminopyridine in hypertensive rats. Eur J Pharmacol 2003;466: 301-310.

16 Yamazaki T, Akiyama T, Kitagawa $\mathrm{H}$, Takauchi Y, Kawada T, Sunagawa K: A new, concise dialysis approach to assessment of cardiac sympathetic nerve terminal abnormalities. Am J Physiol 1997;272:H1182H1187.

17 Minatoguchi S, Majewski H: Milrinone inhibits sympathetic-mediated tachycardia by a postjunctional action independent of cyclic AMP. J Cardiovasc Pharmacol 1991;18:127136.

18 Beavo JA: Cyclic nucleotide phosphodiesterases: functional implications of multiple isoforms. Physiol Rev 1995;75:725-748.

19 Anand-Srivastava MB, Srivastava AK, Cantin M: Pertussis toxin attenuates atrial natriuretic factor-mediated inhibition of adenylate cyclase: involvement of inhibitory guanine nucleotide regulatory protein. J Biol Chem 1987;262:4931-4934.

20 Manganiello VC, Murad F, Vaughan M: Effects of lipolytic and antilipolytic agents on cyclic $3^{\prime}, 5^{\prime}$-adenosine monophosphate in fat cells. J Biol Chem 1971;246:2195-2202.

21 Rump LC, Schuster MJ, Schollmeyer P: Activation of beta 2 -adrenoceptors by isoprenaline and adrenaline enhances noradrenaline release in cortical kidney slices of young spontaneously hypertensive rats. Naunyn Schmiedeberg's Arch Pharmacol 1992;345 25-32.
22 Mulvany MJ, Baumbach GL, Aalkjaer C, Heagerty AM, Korsgaard N, Schiffrin EL, Heistad DD: Vascular remodeling. Hypertension 1996;28:505-506.

23 Rizzoni D, Rodella L, Porteri E, Rezzani R, Guelfi D, Piccoli A, Castellano M, Muiesan ML, Bianchi R, Rosei EA: Time course of apoptosis in small resistance arteries of spontaneously hypertensive rats. J Hypertens 2000;18:885-891.

24 Shakur Y, Holst LS, Landstrom TR, Movsesian M, Degerman E, Manganiello V: Regulation and function of the cyclic nucleotide phosphodiesterase (PDE3) gene family. Prog Nucl Acid Res Mol Biol 2001;66:241-277.

25 Lugnier C: Cyclic nucleotide phosphodiesterase (PDE) superfamily: a new target for the development of specific therapeutic agents. Pharmacol Ther 2006;109:366-398.

26 Mandela P, Ordway GA: The norepinephrine transporter and its regulation. J Neurochem 2006;97:310-333

27 Li Y, Anand-Srivastava MB: Inactivation of enhanced expression of G(i) proteins by pertussis toxin attenuates the development of high blood pressure in spontaneously hypertensive rats. Circ Res 2002;91:247-254.

$\checkmark 28$ Siffert W: G protein polymorphisms in hypertension, atherosclerosis, and diabetes. Annu Rev Med 2005;56:17-28.

29 Judy WV, Watanabe AM, Henry DP, Besch HR Jr, Murphy WR, Hockel GM: Sympathetic nerve activity: role in regulation of blood pressure in the spontaneously hypertensive rat. Circ Res 1976;38:21-29.

30 Rumantir MS, Jennings GL, Lambert GW, Kaye DM, Seals DR, Esler MD: The 'adrenaline hypothesis' of hypertension revisited: evidence for adrenaline release from the heart of patients with essential hypertension. J Hypertens 2000;18:717-723.

$>31$ Whalen EJ, Johnson AK, Lewis SJ: Blockade of beta-adrenoceptors enhances cAMP signal transduction in vivo. Eur J Pharmacol 1998;355:189-193. 